\title{
Stabilization and vibration isolation of a contactless electromagnetic isolator : a frequency-shaped sliding surface approach
}

\author{
Citation for published version (APA): \\ Ding, C., Damen, A. A. H., \& Bosch, van den, P. P. J. (2010). Stabilization and vibration isolation of a \\ contactless electromagnetic isolator : a frequency-shaped sliding surface approach. In Proceedings of the 2010 \\ IEEE/ASME International Conference on Advanced Intelligent Mechatronics (AIM), 6-9 July 2010, Montreal, \\ Ontario (pp. 714-719). Institute of Electrical and Electronics Engineers. \\ https://doi.org/10.1109/AIM.2010.5695866
}

DOI:

10.1109/AIM.2010.5695866

Document status and date:

Published: 01/01/2010

\section{Document Version:}

Publisher's PDF, also known as Version of Record (includes final page, issue and volume numbers)

\section{Please check the document version of this publication:}

- A submitted manuscript is the version of the article upon submission and before peer-review. There can be important differences between the submitted version and the official published version of record. People interested in the research are advised to contact the author for the final version of the publication, or visit the $\mathrm{DOI}$ to the publisher's website.

- The final author version and the galley proof are versions of the publication after peer review.

- The final published version features the final layout of the paper including the volume, issue and page numbers.

Link to publication

\footnotetext{
General rights

- You may freely distribute the URL identifying the publication in the public portal. follow below link for the End User Agreement:

www.tue.nl/taverne

Take down policy

If you believe that this document breaches copyright please contact us at:

openaccess@tue.nl

providing details and we will investigate your claim.
}

Copyright and moral rights for the publications made accessible in the public portal are retained by the authors and/or other copyright owners and it is a condition of accessing publications that users recognise and abide by the legal requirements associated with these rights.

- Users may download and print one copy of any publication from the public portal for the purpose of private study or research.

- You may not further distribute the material or use it for any profit-making activity or commercial gain

If the publication is distributed under the terms of Article $25 \mathrm{fa}$ of the Dutch Copyright Act, indicated by the "Taverne" license above, please 


\title{
Stabilization and Vibration Isolation of a Contactless Electromagnetic Isolator: A Frequency-Shaped Sliding Surface Control Approach
}

\author{
Chenyang Ding, A.A.H. Damen, P.P.J. van den Bosch
}

\begin{abstract}
A Frequency-Shaped Sliding Surface Control (FSSSC) approach is applied to an unstable model of a candidate Electro-Magnetic Isolator (EMI) design which has three Degrees Of Freedom (DOF). The EMI is designed to achieve contactless passive gravity compensation for heavy load by permanent magnets. The 3-DOF model can be regarded as three exactly the same double-integrators disturbed by the nonlinear and coupled passive force which results in its inherent instability. The sliding surface is designed based on relative displacement and payload acceleration feedback to achieve lowfrequency vibration isolation. To avoid the algebraic control loop, a linear converging controller is designed instead of the conventional switching control. Regardless of the plant uncertainties, the closed-loop transmissibility converges to the designed transmissibility with increasing open-loop gain. A sufficient condition for the closed-loop stability is developed. Both time domain and frequency domain performance of the designed controller is evaluated by simulation. It shows that robust vibration isolation performance is achieved despite of the nonlinear and coupled passive force.
\end{abstract}

\section{INTRODUCTION}

In the semiconductor industry, the wafer stepper is one of the core manufacturing machines. One possible bottle neck to further improve the resolution of this machine (currently in the order of $10^{-8} \mathrm{~m}$ ) is the micro-vibrations of a complex lens system which weights a few thousand kilograms. Currently, a six Degrees-Of-Freedom (DOF) suspension system based on three pneumatic isolators [8] is used to support the lens and to reduce the lens vibrations transmitted from the floor or excited by the disturbance forces, including acoustic noises and self-generated forces. The performance of this suspension system is evaluated by two frequency domain criterions. The transmissibility, defined by the transfer function from the floor vibration to the payload vibration, is used to evaluate the vibration isolation performance. The compliance, defined by the transfer function from the force disturbance to the payload vibration, is used to evaluate the disturbance rejection performance. The interested frequency range of both transmissibility and compliance is from zero to hundreds of $\mathrm{Hz}$. In this application, the transmissibility has higher priority because the compliance is already very low due to the huge payload mass. Also, the disturbances can be effectively reduced by other means. For example, a vibration absorber [6] can be attached to the payload to compensate the self-generated disturbance force, vacuum operation can be used to eliminate acoustic noises, etc.

The pneumatic isolators achieve low stiffness and high capacity of gravity compensation by pressurized air. They can be easily stabilized by maintaining the air pressure. However, the high frequency dynamics of the pressurized air is difficult to be accurately modeled or measured so that the control performance is limited. Besides, vacuum operation of the pneumatic isolators would be very difficult.

Contactless gravity compensation in similar scales by passive Permanent Magnetic (PM) force is feasible [9]. To explore this principle, a contactless Electro-Magnetic Isolator (EMI) is being designed [3] as an alternative to the pneumatic isolator. Its two main components, the translator and the stator, have no mechanical contact. Permanent magnets are integrated to produce passive PM force mainly on the vertical direction for gravity compensation and Lorentz actuators are integrated for control. The relation between the PM force and the translator-stator relative position is highly nonlinear and strongly coupled. The nonlinear PM force creates a region wherein the stiffness is very low. It also results in the inherent instability [7] of the EMI. Previous work [3] presents the variation of the PM force with the translatorstator relative position and introduces the 3-DOF model for the EMI. Proper control appoach to simultaneously achieve stabilization and vibration isolation is studied.

Some applications are found to simultaneously achieve stabilization and vibration isolation of a contactless electromagnetic system. In [1], the payload displacement with respect to an inertially fixed reference is used for control. But such signal is not measurable in this application. A 6-DOF suspension system composed of four contactless electromagnetic actuators is built [2] having the gravity compensation capacity of $200 \mathrm{~kg}$. A PI controller is used for stabilization and an $H_{\infty}$ controller is designed for vibration isolation. Simulation [3] shows that such strategy is feasible if the passive PM force is compensated using feedback linearization. But the PM force compensation needs accurate models and it is computationally expensive. Also, the $H_{\infty}$ controller is of high order and the design process is complicated. Therefore, research for an alternative solution is carried out.

L. Zuo and J.J.E. Slotine [4] proposes the FrequencyShaped Sliding Surface Control (FSSSC) approach for active vibration isolation control. According to desired skyhook transmissibility, a sliding surface is designed based on the feedback signals of the relative displacement and absolute velocity. The switching control according to the conventional sliding mode control is applied to guarantee the convergence of the system dynamics to the sliding surface. The resultant performance of the FSSSC approach is robust despite of plant uncertainties and unknown disturbances. However, the dynamics of the velocity sensor (geophone) is not desired. Therefore, industrial acceleration sensors are intended in our 
application. The absolute velocity signal is difficult to obtain due to the limited acceleration sensor performance. Besides, the switching control as in [4] would result in an algebraic control loop due to the acceleration feedback.

This paper investigates stabilization and vibration isolation of the 3-DOF unstable EMI model using the FSSSC approach. Based on the feedback signals of relative displacement and payload acceleration, the sliding surface is designed according to the desired transmissibility and a linear converging controller is designed to realize the designed transmissibility. Closed-loop stability is proved using the small gain theory. The 3-DOF model of the candidate EMI design and the control objective are introduced in Section II. Section III describes the design of the sliding surface and the linear converging controller. The closed-loop stability condition is also developed. Section IV gives the details of the designed controller and evaluates both time domain and frequency domain performance by simulation. This paper is concluded in Section V. In this paper, the subscript $i$ denotes one of the three Cartesian axes $x, y$, and $z$ by default.

\section{PROBLEM STATEMENT}

\section{A. 3-DOF Model of the EMI}

The 3-DOF model of the candidate EMI design is described in [3]. The EMI translator is rigidly fixed to the payload and the mass of this rigid body is denoted by $m$. The payload displacement with respect to an inertially fixed reference is denoted by the vector $\overrightarrow{d_{A}}=\left[d_{A x}, d_{A y}, d_{A z}\right]^{T}$. The EMI stator is rigidly fixed to the vibrating floor. The floor displacement with respect to the same inertially fixed reference is denoted by $\overrightarrow{d_{G}}=\left[d_{G x}, d_{G y}, d_{G z}\right]^{T}$. The payload relative displacement vector is defined as $\overrightarrow{d_{R}}=\overrightarrow{d_{A}}-\overrightarrow{d_{G}}=$ $\left[d_{R x}, d_{R y}, d_{R z}\right]^{T}$. The payload equation of motion is

$$
m \ddot{\overrightarrow{d_{A}}}=\overrightarrow{f_{p}}\left(\overrightarrow{d_{R}}\right)+\overrightarrow{f_{d}}-\vec{u},
$$

where $\overrightarrow{f_{p}}\left(\overrightarrow{d_{R}}\right)=\left[f_{p x}\left(\overrightarrow{d_{R}}\right), f_{p y}\left(\overrightarrow{d_{R}}\right), f_{p z}\left(\overrightarrow{d_{R}}\right)\right]^{T}$ denotes the passive force vector, which is the sum of the PM force and the gravity force. $\overrightarrow{f_{d}}=\left[f_{d x}, f_{d y}, f_{d z}\right]^{T}$ denotes the external disturbance force vector. $\vec{u}=\left[u_{x}, u_{y}, u_{z}\right]^{T}$ denotes the control force vector. Therefore, this 3-DOF model, shown in the dashed rectangular in Fig. 1, can be regarded as three exactly the same double-integrators disturbed by the passive force $\overrightarrow{f_{p}}$. The block $P=\left(m s^{2}\right)^{-1} \cdot I$, where $I$ is the $3 \times 3$ identity matrix. The vector $\overrightarrow{a_{A}}=\left[a_{A x}, a_{A y}, a_{A z}\right]^{T}$ denotes the payload acceleration. The vectors $\overrightarrow{n_{d}}=\left[n_{d x}, n_{d y}, n_{d z}\right]^{T}$ and $\overrightarrow{n_{a}}=\left[n_{a x}, n_{a y}, n_{a z}\right]^{T}$ denote the noises of the three relative displacement sensors and acceleration sensors, respectively. The vectors $\widetilde{\overrightarrow{d_{R}}}=\overrightarrow{d_{R}}+\overrightarrow{n_{d}}$ and $\widetilde{\overrightarrow{a_{A}}}=\overrightarrow{a_{A}}+\overrightarrow{n_{a}}$ denote the outputs of displacement sensors and acceleration sensors, respectively. The vector $\vec{r}=\left[r_{x}, r_{y}, r_{z}\right]^{T}$ denotes the reference.

The point $\overrightarrow{d_{R}}=0$ is chosen as the equilibrium point so that $\overrightarrow{f_{p}}(0)=0$. The working range is defined as the cubic space $\left|d_{R i}\right| \leqslant 1 \mathrm{~mm}$. The entry of $3 \times 3$ stiffness matrix $K, k_{i j}$, is defined as

$$
k_{i j} \triangleq-\frac{\partial f_{p i}}{\partial d_{R j}}, \forall i, j \in\{x, y, z\} .
$$

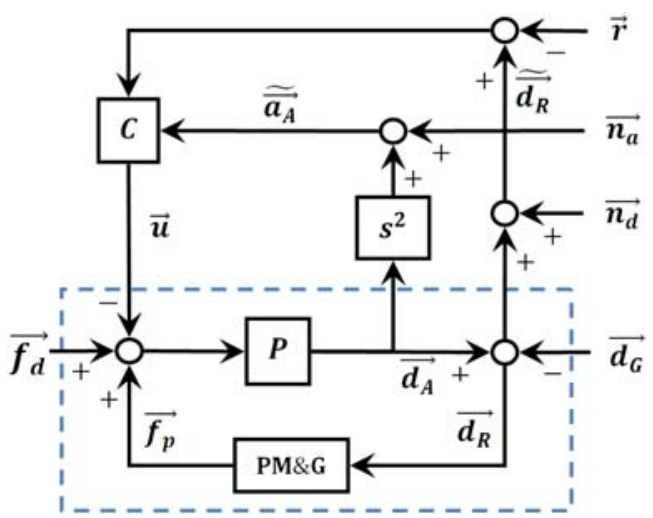

Fig. 1. Control diagram of the nonlinear 3-DOF EMI model. The block $P M \& G$ denotes the permanent magnets and the gravity.

Around the central working range, there is a low-stiffness region, where $\left|k_{i j}\right|, \forall i, j \in\{x, y, z\}$ are very low. But some of $\left|k_{i j}\right|, \forall i, j \in x, y, z$ are comparably very large at the boundary of the working range. Each entry of $K$ has an upper bound and a lower bound within the working range.

For a point $\overrightarrow{d_{R 0}}=\left[d_{R x 0}, d_{R y 0}, d_{R z 0}\right]^{T}$ within the working range, we have $\left|d_{R i 0}\right| \leqslant 1 \mathrm{~mm}$. The Taylor's expansion of $\overrightarrow{f_{p}}\left(\overrightarrow{d_{R}}\right)$ at the point $\overrightarrow{d_{R 0}}$ is given by

$$
\overrightarrow{f_{p}}\left(\overrightarrow{d_{R}}\right) \approx \overrightarrow{f_{p}}\left(\overrightarrow{d_{R 0}}\right)-K\left(\overrightarrow{d_{R}}-\overrightarrow{d_{R 0}}\right) .
$$

Note that the term $\overrightarrow{f_{p}}\left(\overrightarrow{d_{R 0}}\right)+K \overrightarrow{d_{R 0}}$ is a constant force and it does not affect the stability at this point. Therefore, it can be regarded as a part of the disturbance force $\overrightarrow{f_{d}}$. Define vector $\vec{p}=-K \overrightarrow{d_{R}}$, the linearized 3-DOF model can be represented by the dashed rectangular in Fig. 2.

The problem is to design a Double-Input-Single-Output (DISO) controller $C$ for each DOF, as shown in Fig. 1, so that both stabilization and vibration isolation are simultaneously achieved within the working range.

\section{B. Control Objective}

Denote the transmissibility for DOF $i$ by $T_{i}$ and the compliance for DOF $i$ by $C_{p i}$, the objective of the controller design is described as

- The measurements are $\overrightarrow{d_{R}}$ and $\overrightarrow{a_{A}}$.

- Both $T_{i}$ and $C_{p i}$ are stable.

- Interested frequency range: $0-1000 \mathrm{~Hz}$.

- $\left|T_{i}(\omega)\right|=1, \forall \omega \in\left[0, \omega_{r}\right] .\left|T_{i}(\omega)\right|$ decreases with increasing $\omega$ for all $\omega>\omega_{r}$ and the decreasing rate is at least $-40 \mathrm{~dB} /$ dec. $\omega_{r}$ is the resonance frequency of $T_{i}$ and $0<\omega_{r} \leq 1 \mathrm{~Hz}$. Around $\omega_{r},\left|T_{i}(\omega)\right|$ may have a peak but the peak value should be less than $10 \mathrm{~dB}$.

- $\left|C_{p i}(0)\right|=0,\left|C_{p i}(\omega)\right|$ is as low as possible for all $\omega>0$.

The smaller $\omega_{r}$, the better $T_{i}$ in the sense of vibration isolation. However, reducing $\omega_{r}$ increases the probability for the payload and the floor to collide caused by low-frequency $\left(>\omega_{r}\right)$ large-amplitude floor vibrations. Also, lower $\omega_{r}$ demands more accurate low-frequency velocity/acceleration measurement. Currently, the latter factor is the bottle neck for improving $T_{i}$. 


\section{CONTROLLER DESIGN}

\section{A. Background}

The name "frequency-shaped sliding surface" was given by K.D. Young and U. Ozguner [5] in 1993. It is usually applied to tracking control, in which, a measurable signal is to be minimized. The physical interpretation of the frequencyshaped sliding surface to vibration isolation was provided by L. Zuo and J.J.E. Slotine [4] in 2004. In their study, modal decomposition was applied and a sliding surface was designed to achieve skyhook performance for each mode based on absolute velocity feedback. Subsequently, a conventional sliding mode control mechanism, the switching control, was employed as a converging controller to guarantee that the system dynamics converges to the sliding surface.

However, the velocity sensor dynamics is not desired so that acceleration sensors are intended in this application. The absolute velocity signal can not be derived by integration of the acceleration sensor output due to the DC bias. The sliding surface has to be designed based on the relative displacement and payload acceleration feedback. The switching control can not be employed as an converging controller due to the following reasons.

- Even the chatter can be reduced by the boundary layer control [10], the high frequency switching action is difficult for the Lorentz actuator to follow.

- It would result in an algebraic control loop due to the payload acceleration feedback.

- The theoretical compliance does not exist. The theoretical compliance is important because it is difficult to measure due to its extremely low magnitude.

Therefore, the converging controller is designed as a linear controller. The FSSSC approach takes two steps to design the controller $C$ in Fig. 1. The first step is to design the sliding surface for each DOF, described in subsection $B$. The second step is to design a linear converging controller to guarantee that the convergence of the system dynamics, described in subsection $C$. Fig. 2 shows the diagram of the FSSSC approach.

\section{B. Sliding Surface Design}

Based on the feedback signals of the relative displacement $\overrightarrow{d_{R}}$ and the payload acceleration $\overrightarrow{a_{A}}$, the 3-DOF sliding surface is defined by the equation $\vec{\sigma}=0$. The signal $\vec{\sigma}$ is defined by

$$
\vec{\sigma}=\Lambda_{2}(s) \overrightarrow{d_{R}}+\Lambda_{1}(s) \overrightarrow{a_{A}},
$$

where $\Lambda_{1}(s)$ and $\Lambda_{2}(s)$ are two transfer functions to shape the sliding surface. The relation between $\overrightarrow{d_{A}}$ and $\overrightarrow{d_{G}}$ can be obtained by substituting $\overrightarrow{d_{R}}=\overrightarrow{d_{A}}-\overrightarrow{d_{G}}$ and $\overrightarrow{a_{A}}=\overrightarrow{d_{A}} s^{2}$ into the equation $\vec{\sigma}=0$ :

$$
\overrightarrow{d_{A}}\left(\Lambda_{1} s^{2}+\Lambda_{2}\right)=\Lambda_{2} \overrightarrow{d_{G}}
$$

Subsequently, the designed transmissibility for each DOF $T_{d i}$ is derived as

$$
T_{d i}=\frac{D_{A i}(s)}{D_{G i}(s)}=\frac{\Lambda_{2}}{\Lambda_{1} s^{2}+\Lambda_{2}}, \forall i \in\{x, y, z\},
$$

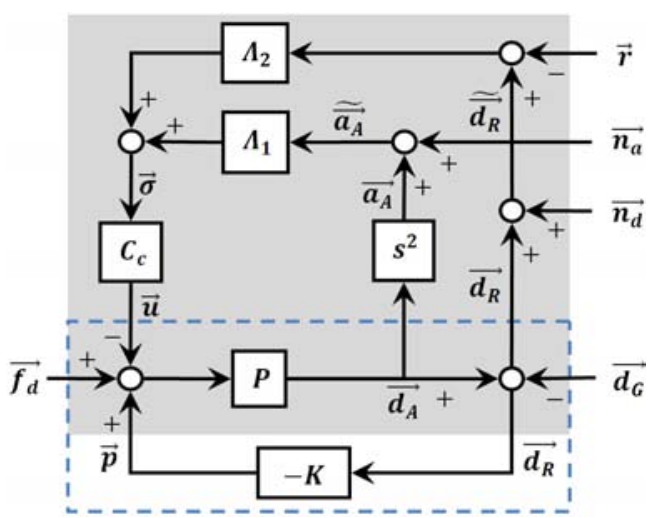

Fig. 2. FSSSC diagram of the linearized 3-DOF EMI model.

where $D_{A i}(s)$ and $D_{G i}(s)$ are the Laplace transforms of the three components of $\overrightarrow{d_{A}}$ and $\overrightarrow{d_{G}}$, respectively. Therefore, based on the requirements of $T_{d i}$, a proper $\Lambda_{1}$ and $\Lambda_{2}$ can be designed according to (6).

The sensor noise should also be considered during the design of $\Lambda_{1}$ and $\Lambda_{2}$. The intended acceleration sensor has a DC bias. Therefore, $\Lambda_{1}$ should have low gain at low frequency and $\Lambda_{1}(0)=0$. The magnitude of $\Lambda_{2}$ is preferred to be low so that the position sensor noise can not be magnified. An example design is given as

$$
\Lambda_{1}=\frac{s\left(s+a_{3}\right)}{s^{2}+b_{1} s+b_{0}}, \quad \Lambda_{2}=\frac{a_{2} s^{2}+a_{1} s+a_{0}}{s^{2}+b_{1} s+b_{0}},
$$

where $b_{0}, b_{1}, a_{0}, a_{1}, a_{2}$, and $a_{3}$ are constants. According to (6), the designed transmissibility would be

$$
T_{d i}=\frac{a_{2} s^{2}+a_{1} s+a_{0}}{s^{4}+a_{3} s^{3}+a_{2} s^{2}+a_{1} s+a_{0}} .
$$

The structure of $T_{d i}$ guarantees that $T_{d i}(0)=1$ and the decreasing rate of $\left|T_{d i}\right|$ at high frequencies is $-40 \mathrm{~dB} / \mathrm{dec}$. The resonance frequency of $T_{d i}$ can be selected by choosing the four stable poles of $T_{d i}$, which determine a unique set of the four parameters $a_{0}, a_{1}, a_{2}$, and $a_{3}$. The denominates of $\Lambda_{1}$ and $\Lambda_{2}$ do not affect the designed transmissibility $T_{d i}$. Based on the noises of both measured signals, two stable poles of both $\Lambda_{1}$ and $\Lambda_{2}$ can be selected. In this way, $b_{0}$ and $b_{1}$ are also uniquely defined.

\section{Convergence Controller Design}

The converging controller $C_{c}$ is used to guarantee that $\vec{\sigma}$ converges to zero regardless of the plant uncertainties. This convergence guarantees the closed-loop stability as long as the sliding surface is stable. To avoid the algebraic control loop, $C_{c}$ can be designed based on the integration of $\vec{\sigma}$.

Define $\Sigma \triangleq \Lambda_{1} s^{2}+\Lambda_{2}$ and treat the block $-K$ as the uncertainty, the closed-loop transmissibility (also named as theoretical transmissibility) $T_{t i}$ are calculated based on the shaded blocks in Fig. 2.

$$
T_{t i}=\frac{P C_{c} \Lambda_{2}}{1+P C_{c} \Sigma}
$$

If $C_{c}$ has such a high gain that $1+P C_{c} \Sigma \approx P C_{c} \Sigma, T_{t i} \approx$ $\frac{P C_{C} \Lambda_{2}}{P C_{C} \Sigma}=T_{d i}$ regardless of the uncertainties in $P$ so that robust 
transmissibility can be achieved. The theoretical compliance $C_{t i}$ are also calculated based on the shaded blocks in Fig. 2 .

$$
C_{t i}=\frac{D_{A i}(s)}{F_{d i}(s)}=\frac{P}{1+P C_{c} \Sigma},
$$

where $F_{d i}(s)$ is the Laplace transform of the three components of the disturbance vector $\overrightarrow{f_{d}}$. Therefore, a controller $C_{c}$ with a higher gain can also reduce $\left|C_{t i}\right|$.

The transfer function from vector $\vec{p}$ to vector $\overrightarrow{d_{R}}$, denoted by $H$, is calculated as

$$
H=\operatorname{diag}\left\{C_{t x}, C_{t y}, C_{t z}\right\} .
$$

Based on the small gain theory, the closed-loop stability is guaranteed if

$$
\|-H K\|_{\infty}<1 .
$$

Therefore, the $C_{c}$ with a higher gain can also improve the robustness of the closed-loop stability. Increasing the open loop gain demands the actuator to have higher force-output capacity. Further more, a high-gain control loop would be more sensitive to the time delay.

\section{PERFORMANCE EVALUATION}

In subsection $A$, the designed sliding surface and $C_{c}$ are given. The simulated time domain and frequency domain performance is described in subsection $B$ and $C$, respectively. The 3-DOF model of the EMI is taken from [3]. The relation between $\|K\|_{\infty}$ and $\overrightarrow{d_{R}}$ within the working range is plotted in Fig. 3. It shows that $\|K\|_{\infty}$ has an upper bound: $\|K\|_{\infty}<$ $2 \times 10^{5}$. The mass of the payload is $m=827.55 \mathrm{~kg}$. For all simulation, the sampling frequency is $10 \mathrm{kHz}$ and a unite delay is added. The resolution of the intended position sensor is $1 \mu \mathrm{m}$ and the resolution of the intended acceleration sensor is $13 \mu \mathrm{m} / s^{2}$. Both sensor noises are simulated by normally distributed random numbers. There are two types of vibration signal used in simulation. The white noise vibration is a set of normally distributed random numbers generated by Simulink and most of the numbers are limited in the range $\pm 0.1 \mathrm{~mm}$. All components of the floor displacement vector $\overrightarrow{d_{G}}$ are simulated by different white noise vibrations. The sweep sine vibration is a sine signal with an amplitude of $0.1 \mathrm{~mm}$ and its frequency varies from 0.1 to $1100 \mathrm{~Hz}$ linearly with the time from 0 to $50 \mathrm{~s}$.

\section{A. Designed Controllers}

The sliding surface is designed as

$$
\Lambda_{1}=\frac{s(s+3)}{s^{2}+\sqrt{2} s+1}, \quad \Lambda_{2}=\frac{3.25 s^{2}+1.5 s+0.25}{s^{2}+\sqrt{2} s+1} .
$$

Therefore, the designed transmissibility is

$$
T_{d i}=\frac{3.25 s^{2}+1.5 s+0.25}{(s+0.5)^{2}(s+1)^{2}} .
$$

The converging controller $C_{c}$ is designed as

$$
C_{c}=5 \times 10^{5} \frac{s+0.5}{s^{2}} .
$$

$\|H\|_{\infty}=\left\|C_{t i}\right\|_{\infty}=2.61 \times 10^{-6}$, the closed-loop stability condition (12) is therefore satisfied. The magnitudes for both $T_{t i}$ and $T_{d i}$ are compared in Fig. 4(a). The two curves coincide

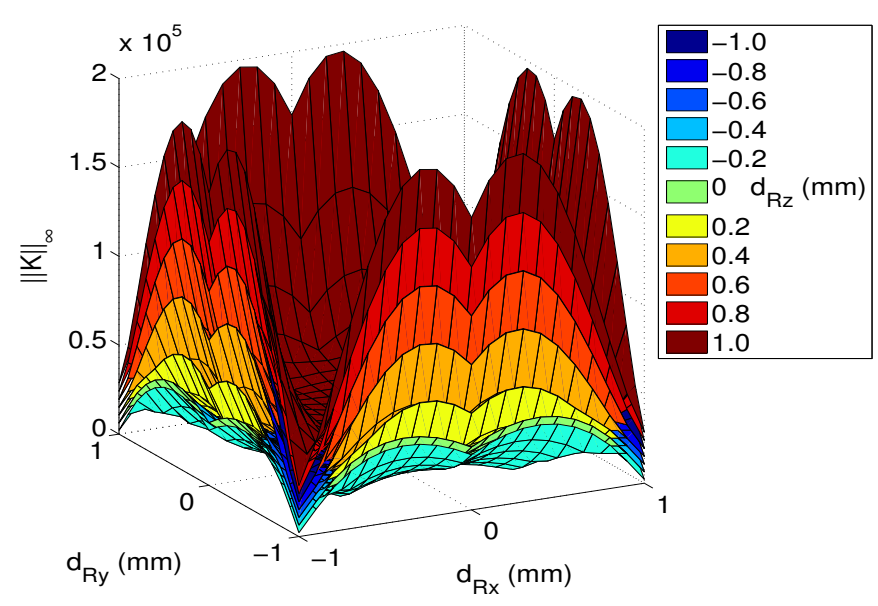

Fig. 3. Variation of $\|K\|_{\infty}$ with the relative displacement vector.

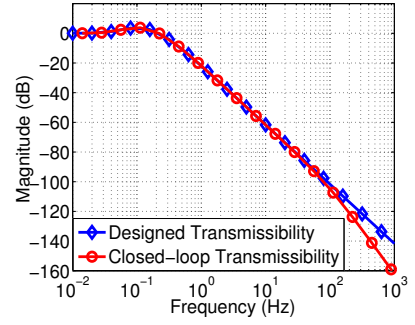

(a) Transmissibility

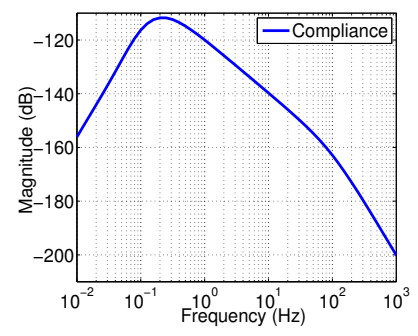

(b) Compliance
Fig. 4. Theoretical frequency domain performance

at low frequencies up to $100 \mathrm{~Hz}$ but $\left|T_{t i}\right|$ decreases faster than $\left|T_{d i}\right|$ at higher frequencies. The theoretical compliance magnitude is plotted in Fig. 4(b).

\section{B. Time Domain Performance}

With white noise floor vibration, the step response is simulated by stepwisely setting the reference vector $\vec{r}$ to $[1,0,1]^{T} \mathrm{~mm}$. To avoid the over-shoot, a pre-filter $F(s)$ is placed between the reference input and the closed-loop system. $F(s)$ is given by

$$
F(s)=\frac{0.038462}{(s+0.5)\left(s^{2}+0.4615 s+0.07692\right)} .
$$

The responses of $\overrightarrow{d_{A}}$ and $\vec{u}$ are shown in Fig. 5(a) and Fig. 5(b), respectively. The three components of $\overrightarrow{d_{A}}$ almost coincide and the steady-state errors converge to zero. By using a pre-filter $F(s)$, the over-shoot is also zero. The control force $\vec{u}$ converges to a constant vector at steady state due to the passive force vector $\overrightarrow{f_{p}}$. The noise of $\vec{u}$ at steady state is caused by the white noise floor vibration.

With white noise floor vibration and zero reference, a constant force of $10 \mathrm{~N}$ is added to the control force $u_{z}$, simulating a stepwise disturbing force on the payload. The transient responses of $\overrightarrow{d_{A}}$ and $\vec{u}$ are plotted in Fig. 6(a) and Fig. 6(b), respectively. The transient peak of $d_{A z}$ is less than $8 \times 10^{-3} \mathrm{~mm}$ and the steady state error converges to zero. The transient response of $\vec{u}$ is extremely fast. It indicates that the closed-loop system is only slightly affected by constant force disturbance. 


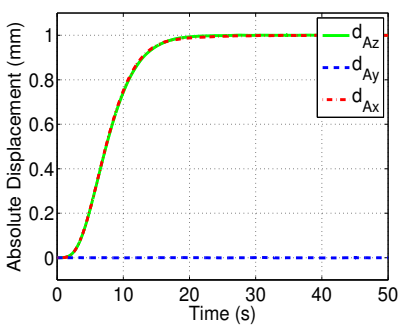

(a) Absolute displacement

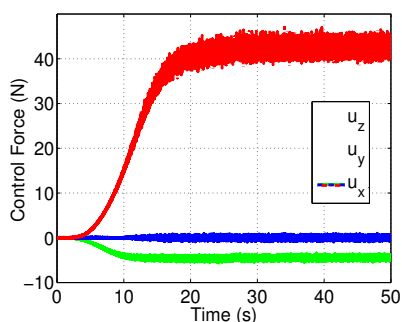

(b) Control force
Fig. 5. Response of tracking a pre-filtered step signal

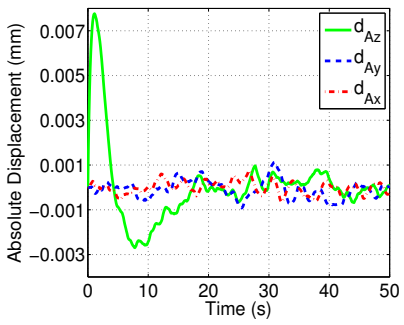

(a) Absolute displacement

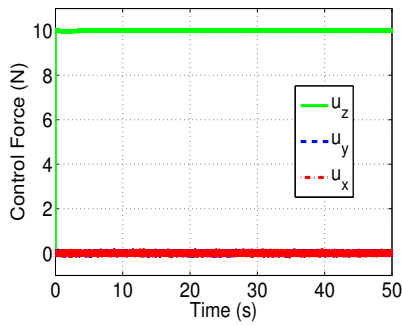

(b) Control force
Fig. 6. Step response to $10 \mathrm{~N}$ vertical constant force disturbance.

With white noise floor vibration and zero reference, a bias of $0.01 \mathrm{~mm} / \mathrm{s}^{2}$ is stepwisely added to each component of the acceleration noise vector $\overrightarrow{n_{a}}$. The transient responses of $\overrightarrow{d_{A}}$ and $\vec{u}$ are plotted in Fig. 7(a) and Fig. 7(b), respectively. As expected, the steady state errors for all components of $d_{A}$ converge to zero, because $\Lambda_{1}(0)$ is designed to be zero.

\section{Frequency Domain Performance}

With zero reference, the response to the sweep sine floor vibration is simulated. The simulated transmissibility $T_{s i}$ are calculated by the Discrete Fourier Transform (DFT) of the absolute displacement $d_{A i}$ over the DFT of the floor displacement $d_{G i}$, shown in Fig. 8. Although the nonlinear and coupled passive force is not considered in the controller design phase, the magnitude curves of $T_{s i}$ are still quite close to that of the theoretical transmissibility. In the frequency range $0.1-10 \mathrm{~Hz}, T_{s i}$ and $T_{t i}$ almost coincide. At higher frequency, $T_{s i}$ are a little noisy, which is caused by the sensor noises. Without the limitation induced by the velocity sensor, $\omega_{r}$ is around $0.1 \mathrm{~Hz}$, which is only one tenth of that in [4].

To test the coupling between the three degrees of freedom

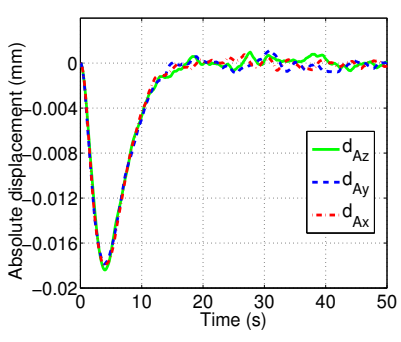

(a) Absolute displacement

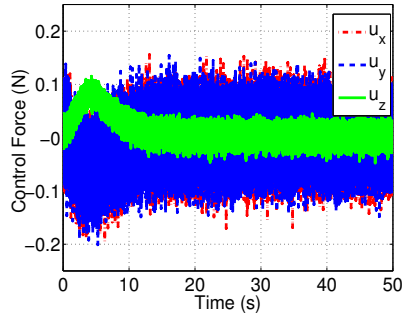

(b) Control force
Fig. 7. Step response to $0.01 \mathrm{~mm} / \mathrm{s}^{2}$ acceleration sensor DC bias.

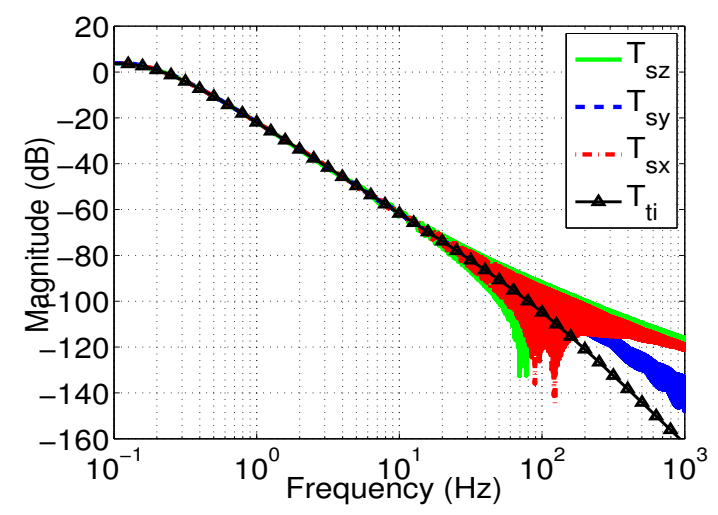

Fig. 8. Simulated transmissibility $T_{s i}$ VS theoretical transmissibility $T_{t i}$.

at the interested frequency range, three processes are simulated. During each process, only one of the three components of $\overrightarrow{d_{G}}$ is excited by the sweep sine vibration. During the process in which $d_{G z}$ is excited, the simulated transmissibility $T_{s i}$ are calculated by the DFT of the absolute displacement $d_{A i}$ over the DFT of the excited floor displacement $d_{G z}$. The simulated transmissibility $T_{s i}$ for this process are plotted in Fig. 9. Similarly, The simulated transmissibility $T_{s i}$ for processes in which $d_{G y}$ or $d_{G x}$ is excited are plotted in Fig. 10 and Fig. 11, respectively. The simulated transmissibility $T_{s i}$ for the un-excited DOF, for example, $T_{s x}$ and $T_{s y}$ in Fig. 9, are not zero. The only two possible causes for this are the sensor noises and the coupled passive force $\overrightarrow{f_{p}}$. To evaluate the influence of the sensor noises, another two processes are simulated. The first process is to keep the floor vibration zero so that all disturbance sources in the closed-loop system are the noises from both type of sensors. The second process is the same as the first process except that the nonlinear passive force is compensated actively which is in parallel with the controller. The influence of the nonlinear passive force would be removed. To make the result comparable to the other processes, the sensor noise transmissibility $T_{n i}$ are calculated by the DFT of $d_{A i}$ over the DFT of the sweep sine vibration and are plotted in Fig. 12.

Fig. 12(a) and 12(b) are almost identical to each other. The $T_{s x}$ in Fig. 9 and Fig. 10 are almost the same as $T_{n x}$ in Fig. 12. It also holds for $T_{s y}$ and $T_{s z}$. It can be concluded that the coupling between any of the two DOF's in frequency range $0.1-1000 \mathrm{~Hz}$ is negligible compared with the influence of the sensor noise. The most probable reason is that all of the five processes are simulated in the low-stiffness region, wherein the variation of the passive force $\overrightarrow{f_{p}}$ is very small.

\section{CONCLUSIONS}

A Frequency-Shaped Sliding Surface Control (FSSSC) approach is proposed for stabilization and vibration isolation of the 3-DOF model of a candidate EMI design. The sliding surface is designed according to the desired transmissibility and the sensor properties based on the feedback signals of relative displacement and payload absolute acceleration. A linear converging controller is designed to guarantee the convergence of the system dynamics. Increasing open-loop 


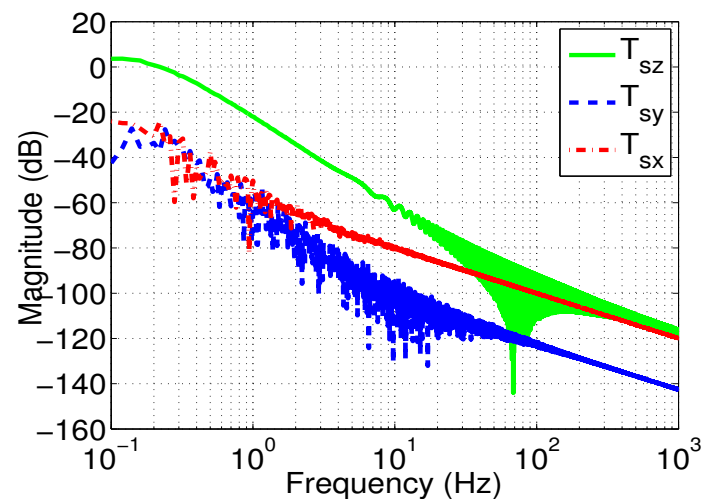

Fig. 9. Simulated transmissibility $T_{s i}$ with only floor vibration $d_{G z}$ excited.

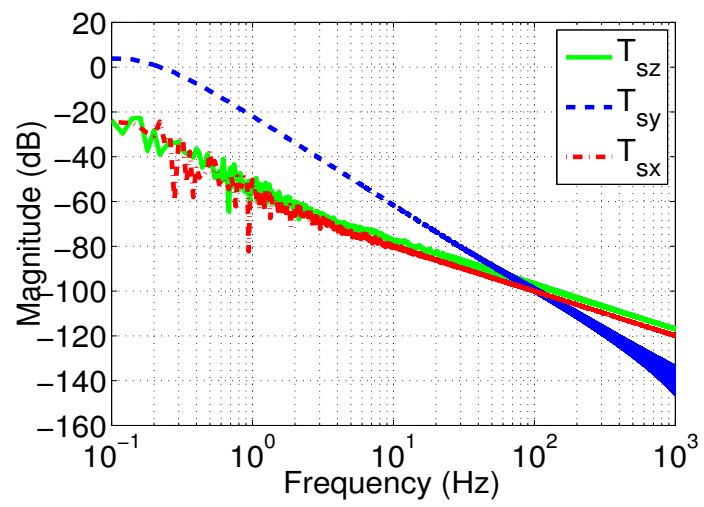

Fig. 10. Simulated transmissibility $T_{s i}$ with only floor vibration $d_{G y}$ excited.

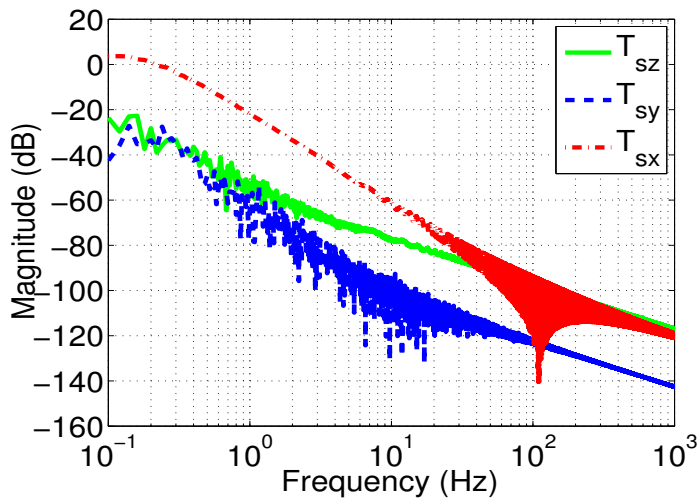

Fig. 11. Simulated transmissibility $T_{s i}$ with only floor vibration $d_{G x}$ excited.

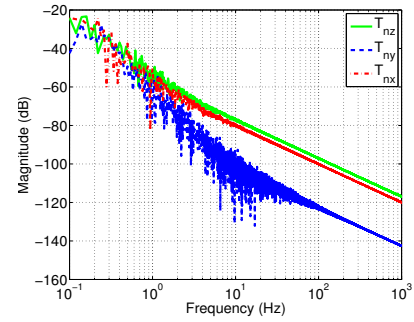

(a) Without force compensation

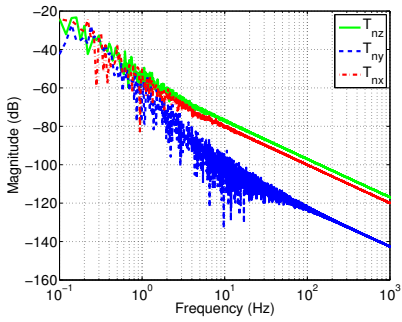

(b) With force compensation
Fig. 12. Comparison of sensor noise transmissibility with and without the influence of the passive force. gain improves the compliance, the stability robustness, and the convergence of the theoretical transmissibility to the designed transmissibility. The bottle neck of the gain rise is the actuator capacity and the time delay of the control loop. Simulation shows that robust performance is achieved within the EMI low-stiffness region although the passive force is not considered in the design phase. The transmissibility resonance frequency is greatly reduced compared to [4]. The simulated transmissibility is very close to the theoretical transmissibility with reasonable sensor noise. The sensor noise has much more influence on transmissibility than the passive force at the low-stiffness region. With a pre-filter, the reference tracking performance is acceptable. Transient response to the stepwise constant force disturbance is excellent. Transient response to the stepwise acceleration sensor bias is also acceptable. The steady state error in all the three time-domain tests converges to zero.

\section{ACKNOWLEDGMENTS}

This work is a part of the Dutch IOP-EMVT program and is supported financially by SenterNovem, an agency of the Dutch Ministry of Economic Affairs.

\section{REFERENCES}

[1] Kosuke Nagaya and Masashi Ishikawa, "A noncontact permanent magnet levitation table with electromagnetic control and its vibration isolation method using direct disturbance cancellation combining optimal regulators", IEEE Trans. on Magnetics, vol. 31, no. 1, Jan. 1995.

[2] Katsuhide WATANABE, Shinji HARA, Yoichi KANEMITSU, Takahide HAGA, Kenichi YANO, Takayuki MIZUNO, and Ryuta KATAMURA, "Combination of $H_{\infty}$ and PI control for an electromagnetically levitated vibration isolation system", Proc. 35th Conf. on Decision and Control, Kobe, Japan, Dec. 1996.

[3] C. Ding, A.A.H. Damen, P.P.J. van den Bosch, and J.L.G. Janssen, "Vibration Isolation of an Electromagnetic Actuator with Passive Gravity Compensation", Proc. 2nd International Conference on Computer and Automation Engineering, Singapore, Feb. 2010.

[4] L. Zuo and J.J.E. Slotine, "Robust vibration isolation via frequencyshaped sliding control and modal decomposition", Journal of Sound and Vibration, vol. 285, no. 4-5, pp. 1123-1149, Aug. 2005.

[5] K.D. Young and U. Ozguner, "Frequency shaping compensator design for sliding mode", International Journal of Control, vol. 57, no. 5, pp. 1005-1019, 1993.

[6] Hakan Elmali, Mark Renzulli, and Nejat Olgac. "Experimental Comparison of Delayed Resonator and PD Controlled Vibration Absorbers Using Electromagnetic Actuators", Transactions of the ASME, vol. 122, pp. 514-520, Sep. 2000.

[7] S. Earnshaw, "On the Nature of the Molecular Forces which Regulate the Constitution of the Luminiferous Ether," Trans. Camb. Phil. Soc., vol. 7, pp. 97-112, 1842.

[8] M. Heertjes, K. de Graaff, and J.G. van der Toorn, "Active Vibration Isolation of Metrology Frames; A Modal Decoupled Control Design", Journal of Sound and Acoustics-Transactions of the ASME, vol. 127, Issue 3, pp. 223-233, Jun. 2005.

[9] Jeroen L. G. JANSSEN, Johannes J. H. PAULIDES and Elena A LOMONOVA, "Passive Limitations for a Magnetic Gravity Compensator", Journal of System Design and Dynamics, vol. 3, no. 4, pp. 671680, 2009

[10] K.D. Young, V.I. Utkin, and U. Ozguner, "A Control Engineer's Guide to Sliding Mode Control", IEEE Trans. on Control Systems Technology, vol. 7, no. 3, pp. 328-342, 1999. 